Sveriges lantbruksuniversitet

Swedish University of Agricultural Sciences

Department of Economics

WORKING PAPER

08/2016

\title{
Rural land development under hyperbolic \\ discounting: a real option approach
}

Luca Di Corato

Economics 


\title{
Rural land development under hyperbolic discounting: a real option approach*
}

\author{
Luca Di Corato ${ }^{\dagger}$
}

\begin{abstract}
This article presents a simple model of land development under uncertainty and hyperbolic discounting. Land kept in rural use pays an uncertain rent, while net returns from land development are known and constant. The landowner is viewed here as a sequence of infinite autonomous selves with time-inconsistent preferences. We solve the underlying noncooperative intra-personal stopping time game under both naïve and sophisticated beliefs about the landowner's time-inconsistency and show that i) land development is accelerated due to his present-biased time preferences and ii) a higher acceleration is associated with sophistication.
\end{abstract}

Keywords: Land Development, Stochastic Hyperbolic Discounting, Timing Game, Real Options.

Jel classification: C61, Q15, R11.

* This paper builds on a frame developed in my $\mathrm{PhD}$ thesis. Comments from Michele Moretto are gratefully acknowledged. The usual disclaimer applies. Last, I gratefully acknowledge financial support from Swedish Research Council FORMAS through the project TRUSTEE.

${ }^{\dagger}$ Corresponding author: Department of Economics, Swedish University of Agricultural Sciences, Box 7013, 75007, Uppsala, Sweden. Email: luca.di.corato@slu.se. Telephone: +46(0)18671758. Fax: +46(0)18673502. 


\section{Introduction}

Use of exponential discounting dominates the literature investigating land development decisions as: i) it lowers the burden in terms of mathematical complexity and, very importantly, ii) it guarantees the time consistency of any development plan set by the landowner due to the underlying assumption of a constant rate of time preference.

However, a constant rate of time preference is a strong assumption that has been hotly debated since Strotz (1956) proposed, as a more "reasonable" alternative, ${ }^{1}$ time-declining discount rates. Strotz's conjecture has since found empirical support, as several experiments have provided evidence of individuals' taste for immediate gratification (see Loewenstein and Prelec, 1992, for a review). This has led to a series of papers using hyperbolic rather than exponential functions in model setup (see Frederick et al., 2002, for a review). Concerning landowners, empirical support for quasihyperbolic discounting has been found by Salois and Moss (2011) in a study based on US farmland values. However, efforts to introduce hyperbolic discounting in the theoretical frame investigating land development decisions have been quite limited and, to our knowledge, are represented solely by the model provided in Salois (2012). Salois introduces a quasi-hyperbolic landowner in the standard model of land development proposed by Irwin and Bockstael (2002) and shows that, by increasing the rate of land conversion, quasi-hyperbolic discounting may induce overdevelopment of rural land.

The analysis in Salois (2012) is performed in a deterministic frame and thus does not permit consideration of the relevant impact that uncertainty about payoffs associated with land uses may have on the decision to develop and on its timing. In fact, as widely recognised in the literature (see for instance Capozza and Helsley, 1990; Capozza and Li, 1994; Geltner et al., 1996), the option value associated with the decision to be taken may make postponing development the optimal choice in order to gather information about its future benefits.

The present study aims to fill this gap by investigating the land development problem under hyperbolic discounting in a real option frame. This enables examination of how two conflicting elements, namely hyperbolic discounting on the one hand, inducing a rush in land development and option value on the other hand inducing delay, affect the decision and timing of land development.

\footnotetext{
${ }^{1}$ Referring to exponential discounting, Strotz (1956) argues that there is "no reason why an individual should have such a special discount function" (p.172).
} 
Since, as noted by Strotz, declining discount rates may lead to time inconsistent planning, the impact of agents' self-awareness about their time inconsistency on the decision and timing of land development is also explicitly investigated. This is done by considering i) a landowner who fully ignores his time inconsistency (naïve) and ii) a landowner who perfectly foresees that his time preferences change over time (sophisticated) (see O'Donoghue and Rabin, 1999).

Previous papers studying real options in the framework of time inconsistent preferences are those by Grenadier and Wang (2007) and Di Corato (2012). Grenadier and Wang (2007) investigate the impact of hyperbolic discounting on investment plans and show that the timing of investment differs from that set by an exponential discounter and depends on: i) agents' self-awareness about their time inconsistency and ii) the payoff form, i.e. a lump sum rather than a sequence of cash flows. Di Corato (2012) considers a forest-cutting problem in a dynamic game played by three nonoverlapping, imperfectly altruistic generations, each of which is viewed as a hyperbolic discounting agent, and shows that, compared with the harvesting policy set by an exponential agent, the option value attached to the decision to harvest is lower and earlier harvest occurs. That result holds under both naïve and sophisticated beliefs but under sophistication harvest occurs earlier than under naïveté.

The present study considers a standard land development problem where land kept in rural use pays a rent following geometric Brownian motion and net returns from land development are known and constant. ${ }^{2}$ In line with the literature, the hyperbolic landowner is viewed as a sequence of infinite autonomous selves with time-inconsistent preferences (see Grenadier and Wang, 2007; Harris and Laibson, 2013). Each self is allowed to exercise the option to develop only during his life and cares about, but has no control over, the decisions taken by his future selves. The life of these selves is random and regulated by a Poisson death process. Each self determines his optimal threshold for exercise of the option to develop by solving the underlying non-cooperative intra-personal stopping game on the basis of his beliefs about the behaviour of future selves.

It is found that a hyperbolic landowner always develops land earlier than an exponential

\footnotetext{
${ }^{2}$ Grenadier and Wang (2007) study the optimal exercise of an American call option on investment under hyperbolic discounting. The present study, in contrast, is dealing with the optimal exercise of an American put-like option to "disinvest", by selling land to a developer. Note also that in our frame holding the option pays a periodic rent (associated with rural use) as a sort of dividend.
} 
landowner. The reasoning is straightforward. Under hyperbolic discounting, the bias for present gratification lowers the value associated with holding the option to develop. Hence, each self finds convenient anticipating future selves by killing the option earlier. Interestingly, this study shows that a sophisticated landowner rushes land development more than a naïve landowner. In fact, due to his perfect foresight, a sophisticated landowner, takes into account the cost of changes in the development timing strategy that are sub-optimal from his time perspective. Hence, compared with a naïve landowner totally ignoring this cost, the sophisticated landowner has a further argument for anticipating future selves by killing the option to develop.

The remainder of this paper is organised as follows. Section 2 presents the basic set-up. In Section 3, the value of land and the timing of land development under hyperbolic discounting are determined, under the assumption of both naïve and sophisticated beliefs. The results are then compared and discussed using the case of an exponential landowner as the benchmark. Section 4 concludes. The Appendix contains the proofs omitted from the text.

\section{The basic set-up}

Consider a risk-neutral, infinite-lived landowner owning a parcel of land currently kept in rural use (agriculture, forestry, open space etc.). Under the current destination, the landowner earns a rent $\{R(t): t \geq 0\}$. Assume that $R(t)$ is stochastic and evolves according to the following diffusion:

$$
d R(t) / R(t)=\alpha d t+\sigma d Q(t), \text { with } R(0)=R
$$

where $\alpha$ and $\sigma$ are the drift and volatility rates, respectively, and $\{Q(t)\}$ is a standard Wiener process with $E[d Q]=0$ and $E\left[(d Q)^{2}\right]=d t$.

At any $t>0$, the landowner can sell his land to a developer, converting it into an urban use. Here it is assumed that land development is irreversible ${ }^{3}$ and the net pay-off ${ }^{4}$ accruing to the landowner selling his land is denoted $P>0 .{ }^{5}$

\footnotetext{
${ }^{3}$ Note that, taking a real options perspective, the landowner can be viewed as holding an American put option, i.e. the option to develop, paying $P$ if exercised. Otherwise, i.e. the land kept in rural use, the landowner receives $R(t)$ as a sort of dividend.

${ }^{4}$ As in Salois (2011), the pay-off $P$ results from the sales price net of any conversion costs (administrative fees, permit expenses, institutional costs or necessary infrastructure expenditures, etc.).

${ }^{5}$ Note that one can easily allow for a stochastic $P$ following geometric Brownian motion. The model would in fact be the same once $R(t)$ is normalised in terms of $P$ (see Geltner et al., 1996, p. 26).
} 


\subsection{The discount function}

The time preferences of a hyperbolic landowner are characterised here by adopting the discount function proposed by Harris and Laibson (2013), where the hyperbolic discounting agent is viewed as an infinite sequence of risk-neutral selves, $i=0,1,2, \ldots$. Each self $i$ divides time into present and future. The present lasts a random time period, $s_{i+1}-s_{i}$, where $s_{i}$ and $s_{i+1}$ are the birth dates of self $i$ and self $i+1$. The transition is regulated by a Poisson death process with intensity $\lambda \in(0, \infty)$. The future, from the perspective of self $i$, starts at $s_{i+1}$ and lasts forever. Each self $i$ discounts 1) exponentially, at a constant rate $\rho$, both present and future payoffs and 2) additionally, by the factor $\beta \in(0,1)$ all future payoffs. Summing up, his discount function is defined as follows:

Definition 1 At time $t$ for any $\beta \in(0,1)$ and $\lambda \in(0, \infty)$, the self $i^{\prime} s$ discount function is given by the following function:

$$
\begin{aligned}
D_{i}(s, t) & =\left\{\begin{array}{l}
e^{-\rho(t-s)}, \quad \text { if } t \in\left[s_{i}, s_{i+1}\right), \\
\beta e^{-\rho(t-s)}, \quad \text { if } t \in\left[s_{i+1}, \infty\right),
\end{array}\right. \\
\text { for } t & >s \text { and } s_{i} \leq s \leq s_{i+1} .
\end{aligned}
$$

From Eq. (2), as $\beta \in(0,1)$, a dollar paid to the generic self $i$ in the future would be worth less than a dollar paid in the present. Note that in the limit cases $\beta \rightarrow 1$ and $\beta \rightarrow 0$, the agent would discount exponentially at rate $\rho$ and $\rho+\lambda$, respectively, where $\lambda$ is the "speed" at which time preferences change over time. ${ }^{6}$ Note also that:

i) The expected value of $D_{i}(s, t)$, i.e.

$$
E\left[D_{i}(s, t)\right]=e^{-(\rho+\lambda)(t-s)}+\beta\left(1-e^{-\lambda(t-s)}\right) e^{-\rho(t-s)}=\beta e^{-\rho(t-s)}+(1-\beta) e^{-(\rho+\lambda)(t-s)},
$$

is a convex combination of two exponential discount functions using $\rho$ and $\rho+\lambda$ as discount rates.

ii) The derivative of $E\left[D_{i}(s, t)\right]$ with respect to time is:

$$
\left.\partial E\left[D_{i}(s, t)\right] / \partial t=-\left[\rho \beta e^{-\rho(t-s)}+(\rho+\lambda)(1-\beta) e^{-(\rho+\lambda)(t-s)}\right\}\right]<0 .
$$

\footnotetext{
${ }^{6}$ The probability of having a new self born in the next time interval $d t$ is equal to $\lambda d t$. Hence, consistently, when $\beta \rightarrow 0$ the discount rate is adjusted in order to account for the "sudden death" of the current self.
} 
This implies that the discount factor increases as $t$ comes nearer or that, in other words, payoffs are discounted at the following time-declining rate:

$$
-\left(\partial E\left[D_{i}(s, t)\right] / \partial t\right) / E\left[D_{i}(s, t)\right]=\rho+\lambda(1-\beta) e^{-(\rho+\lambda)(t-s)} / E\left[D_{i}(s, t)\right]
$$

\section{Value of land and timing of land development}

In order to provide a benchmark, in this section the standard land development problem faced by a landowner who discounts future payoffs exponentially is first solved. A hyperbolic landowner with the time preferences presented above is then considered. This landowner discounts future payoffs at time-declining rates and may formulate time-inconsistent plans, i.e. he may formulate plans which may later be disobeyed and revised. The analysis then proceeds by allowing for two extreme assumptions concerning the landowner's self-awareness about his time-inconsistency. First a naïve agent, i.e. an agent who ignores his time inconsistency and believes that his future selves will act according to his current time preferences, is considered. A sophisticated agent, i.e. an agent who perfectly foresees that his time preferences change over time, is then considered. Under nä̈veté, the timing strategy set by the current self will be formulated viewing future selves as "committed" to his plan of action. Under sophistication, in contrast, the current self sets the strategy, being aware that future selves are going to "disobey". ${ }^{7}$ The resulting timing strategy then takes into account the sub-optimality, from the present self's time perspective, associated with the timing strategies set by his future selves.

\subsection{An exponential landowner}

The landowner discounts exponentially future payoffs ${ }^{8}$ at the constant discount rate $\rho>\alpha .^{9}$ Assume that it is currently worth keeping land in rural use, i.e. $R>R^{*}$ where $R^{*}$ is the revenue threshold triggering land development.

\footnotetext{
${ }^{7}$ The present study focuses on "consistent planning", i.e. the agent do not choose plans that are going to be disobeyed. It does not consider the alternative possibility of selecting a "strategy of precommitment" which would require committing to a certain plan of action (see e.g Pollak, 1968; Strotz, 1956).

${ }^{8}$ The analysis in this section is consistent with the limit case where $\beta \rightarrow 1$ in Eq. (2).

${ }^{9}$ This restriction is needed in order to ensure convergence. Note that if $\rho \leq \alpha$ land development would be never optimal.
} 
Using standard arguments, in the continuation region $R>R^{*}$, the value of land, $V(R)$, is the solution of the following problem:

$$
V(R)=\max _{R}\left\{P, R d t+e^{-\rho d t} E[V(R+d R)]\right\}
$$

By Ito's lemma, Eq. (3) can be rearranged as:

$$
(1 / 2) \sigma^{2} R^{2} V^{\prime \prime}(R)+\alpha R V^{\prime}(R)-\rho=-R, \text { for } \quad R>R^{*} .
$$

Solving Eq. (3.1) yields the following proposition:

Proposition 1 Under exponential discounting,

i) land development occurs when revenues from rural land use reach the threshold

$$
R^{*}=[\gamma /(\gamma-1)] P(\rho-\alpha)
$$

ii) the value of land is equal to:

$$
V\left(R ; R^{*}\right)=\left[P-R^{*} /(\rho-\alpha)\right]\left(R / R^{*}\right)^{\gamma}+R /(\rho-\alpha),
$$

where $\gamma$ is the negative root of the characteristic equation $\Lambda(\gamma)=(1 / 2) \sigma^{2} \gamma(\gamma-1)+\alpha \gamma-\rho=0$.

Proof. See section A.1 in Appendix.

Examining the threshold in Eq. (4), it can be seen that: ${ }^{10}$ i) the higher the expected growth rate, $\alpha$, for returns from rural uses, the lower the critical threshold and the later, in expected terms, land is converted in urban use, i.e. $\partial R^{*} / \partial \alpha<0$; ii) as returns from rural uses become more volatile, the critical threshold is lowered and land development is postponed, i.e. $\partial R^{*} / \partial \sigma^{2}>0$; iii) the higher the discount rate, the earlier land is converted, i.e. $\partial R^{*} / \partial \rho>0$; and iv) the higher the future net returns from urban use, the earlier land is converted, i.e. $\partial R^{*} / \partial P>0$.

In Eq. (5), the first term represents the value of the option to develop land, i.e. the net returns from development, $P$, minus the present value of forgone returns from rural land use, $R^{*} /(\rho-\alpha)$. Note that this payoff materialises only if $R$ reaches $R^{*}$. Hence, consistently, the payoff is discounted using the stochastic discount factor $\left(R / R^{*}\right)^{\beta}$ which accounts for the probability of hitting $R^{*}$. The

\footnotetext{
${ }^{10}$ These results are pretty standard in the literature, see for instance Capozza and Helsley (1990) and Capozza and Li (1994).
} 
second term in Eq. (5) is the expected present value of returns from rural land use. This would be the value accruing to the landowner if the condition triggering land development were not achieved. In this respect, note that as $R^{*} \rightarrow 0$, the value of the option to develop vanishes as, by Eq. (1), $R(t)>0$ and $\lim _{R^{*} \rightarrow 0} V\left(R ; R^{*}\right)=R /(\rho-\alpha)$.

\subsection{A hyperbolic naïve landowner}

The present self $i$ discounts by $e^{-\rho d t}$ any payoff accruing in the time interval $\left(s, s_{i+1}\right)$ and by

$\beta e^{-\rho d t}$ the pay-offs accruing in the interval $\left[s_{i+1}, \infty\right)$. Under nä̈veté, self $i$ believes that the entire sequence of successive selves, i.e. selves in the interval $\left[s_{i+1}, \infty\right)$, will take decisions concerning the destination of land according to his own time preferences, i.e. $D_{i}(s, t)$. This implies that self $i$ views these selves as standard exponential discounters using $\rho$ as discount rate and having the following plan of action:

$$
\begin{array}{ll}
\text { keep land in rural use, } & \text { if } R>R^{*}, \\
\text { sell land, } & \text { if } R \leq R^{*} .
\end{array}
$$

Self $i$ solves the land development problem on the basis of his beliefs and sets $R^{n}$ as the critical threshold. If $R(t)$ hits $R^{n}$ before the next self is born, self $i$ cashes the flow of rents accruing up to the land sale plus the sale net pay-off $P$. Otherwise, if self $i+1$ is born before $R(t)$ hits $R^{n}$, self $i$ benefits from the flow of rents accruing in the period $\left(s, s_{i+1}\right)$ plus the expected present value of the pay-offs cashed by future selves. Consistently with his beliefs, i.e. future selves developing at $R^{*}$, this continuation value is equal to:

$$
V_{c}^{n}\left(R ; R^{*}\right)=\beta \cdot V\left(R ; R^{*}\right)
$$

Assume that it is currently worth keeping land in rural use, i.e. $R>R^{n}$. The present self $i$ must then solve the following problem:

$$
V^{n}(R)=\max _{R}\left\{P, R d t+e^{-\lambda d t} E\left[e^{-\rho d t} V^{n}(R+d R)\right]+\left(1-e^{-\lambda d t}\right) E\left[e^{-\rho d t} V_{c}^{n}\left(R+d R ; R^{*}\right)\right]\right\}
$$

By Ito's lemma, Eq. (6) can be rearranged as:

$$
(1 / 2) \sigma^{2} R^{2} V^{n \prime \prime}(R)+\alpha R V^{n \prime}(R)-\rho V^{n}(R)=-\left\{R+\lambda\left[V_{c}^{n}\left(R ; R^{*}\right)-V^{n}(R)\right]\right\} .
$$

Solving Eq. (6.1) yields the following proposition: 
Proposition 2 Under hyperbolic discounting and naïve beliefs,

i) the land development threshold $R^{n}$ is the solution of the following equation:

$$
\beta[(\theta-\gamma) /(\theta-1)]\left[P-R^{*} /(\rho-\alpha)\right]\left(R^{n} / R^{*}\right)^{\gamma}+\eta\left[R^{n} /(\rho-\alpha)\right]-[\theta /(\theta-1)] P=0
$$

ii) land is, in expected terms, developed earlier than under exponential discounting, i.e. $R^{n}>$ $R^{*}$;

iii) the value of land is equal to:

$$
\begin{gathered}
V^{n}\left(R ; R^{n}, R^{*}\right)=\left\{P-\beta\left[P-R^{*} /(\rho-\alpha)\right]\left(R^{n} / R^{*}\right)^{\gamma}-\eta\left[R^{n} /(\rho-\alpha)\right]\right\}\left(R / R^{n}\right)^{\theta}+ \\
+\beta\left[P-R^{*} /(\rho-\alpha)\right]\left(R / R^{*}\right)^{\gamma}+\eta[R /(\rho-\alpha)], \text { for } R>R^{n},
\end{gathered}
$$

where $\eta=(\rho+\lambda \beta-\alpha) /(\rho+\lambda-\alpha) \leq 1$ and $\theta$ is the negative root of the characteristic equation $\Omega(\theta)=(1 / 2) \sigma^{2} \theta(\theta-1)+\alpha \theta-(\rho+\lambda)=0$.

Proof. See section A.2 in Appendix.

The critical threshold $R^{n}$ set by a hyperbolic nä̈ve landowner is higher than that set by an exponential landowner. This implies that, in expected terms, land is developed earlier. This makes sense considering that, due to his present-biased time preferences, the value of keeping open the option to develop has lower value for self $i$. Note in fact that the value associated with this option when held by his future selves, i.e. the flow of rents accruing up to the land sale plus the sale net pay-off $P$, is lowered by the terms $\eta<1$ and $0<\beta<1$, respectively. Hence, the optimal timing strategy for self $i$ is anticipating his future selves by setting a higher threshold for exercise of the option to develop. Note that $d R^{n} / d \beta<0$ and $d R^{n} / d \lambda>0$. This means that the higher the present bias, i.e. the lower $\beta$ and/or the higher $\lambda$, the earlier land development occurs.

Finally, note that the timing strategy set by a hyperbolic naïve landowner is based on the belief that his future selves are committed to S.1, i.e. the timing strategy set by the current self. This belief is, of course, unfounded. In fact, as soon as the next self $i+1$ is born, he will not adopt the threshold $R^{*}$ as believed by self $i$, but will set his timing strategy on the basis of his own time preferences, i.e. using $D_{i+1}(s, t)$. Given that, by assumption, the sequence of selves is infinite and naïveté persistent, each newly born self faces the same decision problem solved above for self $i$. Hence, it can be concluded that the actual critical threshold for exercise of the option to develop land is unique and equal to $R^{n}>R^{*}$. 


\subsection{A hyperbolic sophisticated landowner}

A sophisticated self $i$ has perfect foresight and anticipates that his future selves may later revise his plan of action. Hence, his plan should be set taking into account that, from his time perspective, future selves' timing strategies may be sub-optimal. In the present frame, the landowner is viewed as a sequence of infinite selves each having their own time preferences, i.e. $D_{i}(s, t)$. However, as each self is followed by an infinite number of selves, the land development problem that each generic self must solve is the same. Hence, as they would all consider optimal developing land when the same critical time threshold is reached, their timing strategy can be determined by imposing stationarity to the solution of the underlying non-cooperative intra-personal timing game.

Consider the generic self $i$ and denote i) by $\widetilde{R}$ his conjecture about the critical development threshold set by his future selves and ii) by $g(\widetilde{R})$ his own critical development threshold. Note that, as his timing strategy must be consistent with his beliefs, the threshold $g(\widetilde{R})$ is a function of the conjectured threshold $\widetilde{R}$.

Future selves have the following plan of action:

$$
\begin{array}{ll}
\text { keep land in rural use, } & \text { if } R>\widetilde{R}, \\
\text { sell land, } & \text { if } R \leq \widetilde{R} .
\end{array}
$$

Hence, as above, given S.2 and his time perspective, self $i$ 's continuation value is equal to:

$$
V_{c}^{s}(R ; \widetilde{R})=\beta\left\{[P-\widetilde{R} /(\rho-\alpha)](R / \widetilde{R})^{\gamma}+R /(\rho-\alpha)\right\}
$$

Assume that $R>g(\widetilde{R})$. The present self $i$ must then solve the following problem:

$$
V^{s}(R)=\max _{R}\left\{P, R d t+e^{-\lambda d t} E\left[e^{-\rho d t} V^{s}(R+d R)\right]+\left(1-e^{-\lambda d t}\right) E\left[e^{-\rho d t} V_{c}^{s}(R+d R ; \widetilde{R})\right]\right\}
$$

By Ito's lemma, Eq. (9) can be rearranged as:

$$
(1 / 2) \sigma^{2} R^{2} V^{s \prime \prime}(R)+\alpha R V^{s \prime}(R)-\rho V^{s}(R)=-\left\{R+\lambda\left[V_{c}^{s}(R ; \widetilde{R})-V^{s}(R)\right]\right\} .
$$

Solving Eq. (9.1) gives:

$$
\begin{gathered}
V^{s}(R ; h(\widetilde{R}), \widetilde{R})=\left\{P-\beta[P-\widetilde{R} /(\rho-\alpha)](g(\widetilde{R}) / \widetilde{R})^{\gamma}-\eta[g(\widetilde{R}) /(\rho-\alpha)]\right\}(R / g(\widetilde{R}))^{\theta}+ \\
+\beta[P-\widetilde{R} /(\rho-\alpha)](R / \widetilde{R})^{\gamma}+\eta[R /(\rho-\alpha)], \text { for } R>g(\widetilde{R}),
\end{gathered}
$$


where $g(\widetilde{R})$ is the solution of the following equation:

$$
\beta[(\theta-\gamma) /(\theta-1)][P-\widetilde{R} /(\rho-\alpha)](g(\widetilde{R}) / \widetilde{R})^{\gamma}+\eta[g(\widetilde{R}) /(\rho-\alpha)]-[\theta /(\theta-1)] P=0
$$

By imposing the stationarity condition $g(\widetilde{R})=\widetilde{R}=R^{s}$, it can be shown that:

Proposition 3 Under hyperbolic discounting and sophisticated beliefs,

i) the land development threshold is

$$
R^{s}=\frac{\theta+\beta(\gamma-\theta)}{\eta(\theta-1)+\beta(\gamma-\theta)} P(\rho-\alpha)
$$

ii) land is, in expected terms, developed earlier than under naïve beliefs, i.e. $R^{s}>R^{n}$, and

$$
R^{*}<R^{s}<R^{* *}=[\theta /(\theta-1)] P(\rho+\lambda-\alpha)
$$

iii) the value of land is equal to:

$$
\begin{gathered}
V\left(R ; R^{s}\right)=(1-\beta)\left\{\left[P-R^{s} /(\rho+\lambda-\alpha)\right]\left(R / R^{s}\right)^{\theta}+[R /(\rho+\lambda-\alpha)]\right\}+ \\
+\beta\left\{\left[P-R^{s} /(\rho-\alpha)\right]\left(R / R^{s}\right)^{\gamma}+[R /(\rho-\alpha)]\right\}, \text { for } R>R^{s}
\end{gathered}
$$

Proof. See sections A.2 and A.3 in Appendix.

The critical threshold, $R^{s}$, is increasing in the degree of present bias, i.e. $d R^{s} / d \beta<0$ and $d R^{s} / d \lambda>0$. In the limit cases $\beta \rightarrow 1$ and $\beta \rightarrow 0$, the threshold converges toward $R^{*}$ and $R^{* *}$, i.e. the critical thresholds that would be set by an exponential discounter using $\rho$ and $\rho+\lambda$ as discount rates, respectively. The threshold $R^{s}$ is higher than $R^{*}$. As for the case of a naïve landowner, this is again due to the need for avoiding, by killing the option, the passage of self $i$ 's holdings, i.e. the flow of rents plus the option to develop, to future selves. In this respect, note that under sophistication the incentive for anticipating future selves is even higher and leads to the definition of a threshold $R^{s}$ higher than that set by a naïve landowner. This makes the actual exercise by self $i$ of the option to develop even more likely than under naïveté. The reasoning behind this result is straightforward. Self $i$ in fact, being aware of his time inconsistency, fully internalises the cost of sub-optimal (from his time perspective) timing strategies set by his future selves. This cost makes the value associated with holding the option to develop even lower than for the case of a naïve landowner. Last, the threshold $R^{s}$ is lower than $R^{* *}$, i.e. the threshold corresponding to the case 
where the arrival of the next self would, as $\beta \rightarrow 0$, substantially absorb any value associated with self $i$ 's holdings.

Finally, the value function in Eq. (11) results from the weighted sum of the two value functions that an exponential discounter would have using $\rho+\lambda$ and $\rho$ as discount rates, respectively, and fixing $R^{s}$ as the threshold for exercise of the option to develop. This is of course consistent, as clearly illustrated by Eq. (2.1), with the expected discount rate associated with the time preferences of the sophisticated hyperbolic agent considered.

\section{Conclusions}

This paper examines the implications of hyperbolic time preferences for the timing of development of rural land. It extends the previous model by Salois (2012) in order to account for i) uncertainty about payoffs associated with the asset land and ii) self-awareness about the planning time inconsistency implied by the assumed time preferences. The results show that the impact of option value considerations leading, as uncertainty increases, to the postponement of land development is lower under hyperbolic time preferences. In fact, due to the present bias induced by the implicit time-declining discount rate, the value associated with holding open the option to develop is lower and, as a consequence, the hyperbolic landowner prefers earlier land development than in the case of an exponential landowner. It is found that a sophisticated hyperbolic landowner sets a higher threshold for exercise of the option to develop compared with a naïve landowner. In fact, a sophisticated landowner, being aware that the plan initially set may be later revised, sets his timing strategy by fully internalising the cost associated with the sub-optimality of future changes in the strategy. In contrast, this cost is totally missed by a naïve landowner, who believes that the development timing strategy initially set would be not violated. Hence, a further argument rushing land development emerges under sophistication.

These findings refer to land development problems, but may also prove useful for other issues characterising land-use change, such as farmland abandonment, land conservation and competition for land for cultivation of food or energy crops. ${ }^{11}$

\footnotetext{
${ }^{11}$ See Nishihara (2002), Schatzki (2003) and Song et al. (2011), respectively.
} 


\section{Appendix}

\section{A.1 Exponential discounting}

As standard, to guarantee optimality, ${ }^{12}$ the solution of the differential Eq. (3.1) must meet the following value-matching and smooth-pasting conditions:

$$
V\left(R^{*}\right)=P, V^{\prime}\left(R^{*}\right)=0 .
$$

A candidate solution for Eq. (3.1) takes the form: ${ }^{13}$

$$
V(R)=k R^{\gamma}+R /(\rho-\alpha)
$$

where $k$ is a constant to be determined and $\gamma$ is the negative root of the characteristic equation $\Lambda(\gamma)=(1 / 2) \sigma^{2} \gamma(\gamma-1)+\alpha \gamma-\rho=0$.

Substituting Eq. (A.1.3) into Eq. (A.1.1) and Eq. (A.1.2) yields:

$$
\left\{\begin{array}{c}
k R^{* \gamma}+R^{*} /(\rho-\alpha)=P, \\
k \gamma R^{* \gamma-1}+1 /(\rho-\alpha)=0 .
\end{array}\right.
$$

Solving this system gives $k$ and $R^{*}$. Plugging $k$ into Eq. (A.1.3) yields Eq. (5).

\section{A.2 Hyperbolic discounting}

Eq. (6.1) and Eq. (9.1) are technically similar. Proceed by solving first the underlying common problem and then characterising the solution according to the assumed beliefs concerning future selves' time preferences.

The equation to be solved is:

$$
\begin{aligned}
& (1 / 2) \sigma^{2} R^{2} V^{q \prime \prime}(R)+\alpha R V^{q \prime}(R)-(\rho+\lambda) V^{q}(R) \\
& =-\left\{R[1+\lambda \beta /(\rho-\alpha)]+\lambda \beta\left\{\left[P-\bar{R}^{q} /(\rho-\alpha)\right]\left(R / \bar{R}^{q}\right)^{\gamma}\right\}\right.
\end{aligned}
$$

\footnotetext{
${ }^{12}$ See Dixit and Pindyck (1994, Ch. 4).

${ }^{13}$ The solution for the homogeneous part of Eq. (3.1) should have the form $V_{h}(R)=k_{1} R^{\gamma_{1}}+k_{2} R^{\gamma_{2}}$ where $k_{1}$ and $k_{2}$ are constants to be determined while $\gamma_{1}>0$ and $\gamma_{2}<0$ are the roots of the characteristic equation $\Lambda(\gamma)=0$. However, as $R \rightarrow \infty$, the value of the option to develop goes to zero. Thus, as $\gamma_{1}>0$, then $k_{1}$ must be zero, otherwise $\lim _{R \rightarrow \infty} V_{h}(R)=\infty$.
} 
where

$$
\begin{array}{ll}
q=n \text { and } \bar{R}^{n}=R^{*}, & \text { if the agent is naïve, } \\
q=s \text { and } \bar{R}^{s}=\widetilde{R}, \quad \text { if the agent is sophisticated. }
\end{array}
$$

Suppose that the particular solution for Eq. (A.2.1) takes the form $V^{q}(R)=c_{1} R^{\gamma}+c_{2} R$. Substituting this candidate form and its first two derivatives, $\partial V^{q}(R) / \partial R=c_{1} \gamma R^{\gamma-1}+c_{2}$ and $\partial^{2} V^{q}(R) / \partial R^{2}=$ $c_{1} \gamma(\gamma-1) R^{\gamma-2}$ into Eq. (A.2.1) yields:

$$
\begin{aligned}
& \left\{\left[(1 / 2) \sigma^{2} \gamma(\gamma-1)+\alpha \gamma-\rho\right]-\lambda\right\} c_{1} R^{\gamma}+[\alpha-(\rho+\lambda)] c_{2} R \\
& =-\left\{R[1+\lambda \beta /(\rho-\alpha)]+\lambda \beta\left\{\left[P-\bar{R}^{q} /(\rho-\alpha)\right]\left(R / \bar{R}^{q}\right)^{\gamma}\right\}\right.
\end{aligned}
$$

The coefficients $c_{1}$ and $c_{2}$ can be determined by solving the following two equations:

$$
\begin{aligned}
\left\{\left[(1 / 2) \sigma^{2} \gamma(\gamma-1)+\alpha \gamma-\rho\right]-\lambda\right\} c_{1} & =-\lambda \beta\left[P-\bar{R}^{q} /(\rho-\alpha)\right]\left(1 / \bar{R}^{q}\right)^{\gamma} \\
{[\alpha-(\rho+\lambda)] c_{2} } & =-[1+\lambda \beta /(\rho-\alpha)]
\end{aligned}
$$

Solving both equations yields:

$$
\begin{aligned}
& c_{1}=\beta\left[P-\bar{R}^{q} /(\rho-\alpha)\right]\left(1 / \bar{R}^{q}\right)^{\gamma} \\
& c_{2}=[1+\lambda \beta /(\rho-\alpha)] /(\rho+\lambda-\alpha)=\eta /(\rho-\alpha)
\end{aligned}
$$

where $\eta=(\rho+\lambda \beta-\alpha) /(\rho+\lambda-\alpha) \leq 1$.

The general solution then takes the form: ${ }^{14}$

$$
V^{q}\left(R ; \bar{R}^{q}\right)=k R^{\theta}+\beta\left[P-\bar{R}^{q} /(\rho-\alpha)\right]\left(R / \bar{R}^{q}\right)^{\gamma}+\eta[R /(\rho-\alpha)]
$$

where $k$ is a constant to be determined and $\theta$ is the negative root of the characteristic equation $\Omega(\theta)=(1 / 2) \sigma^{2} \theta(\theta-1)+\alpha \theta-(\rho+\lambda)=0$.

At the critical threshold value, $g\left(\bar{R}^{q}\right)$, optimality requires that the following value-matching and smooth-pasting conditions hold:

$$
V^{q}\left(R^{q} ; \bar{R}^{q}\right)=P, \quad \partial V^{q}\left(R ; \bar{R}^{q}\right) /\left.\partial R\right|_{R=g\left(\bar{R}^{q}\right)}=0
$$

\footnotetext{
${ }^{14}$ The solution for the homogeneous part of Eq. (A.2.1) should have the form $V_{h}^{q}(R)=k_{1} R^{\theta_{1}}+k_{2} R^{\theta_{2}}$ where $k_{1}$ and $k_{2}$ are constants to be determined while $\theta_{1}>0$ and $\theta_{2}<0$ are the roots of the characteristic equation $\Omega(\theta)=0$. However, as $R \rightarrow \infty$, the value of the option to develop goes to zero. Thus, as $\theta_{1}>0$, then $k_{1}$ must be zero, otherwise $\lim _{R \rightarrow \infty} V_{h}^{q}(R)=\infty$.
} 
Solving the system [A.2.4-A.2.5] yields:

$$
k=\left\{P-\beta\left[P-\bar{R}^{q} /(\rho-\alpha)\right]\left(g\left(\bar{R}^{q}\right) / \bar{R}^{q}\right)^{\gamma}+\eta\left[g\left(\bar{R}^{q}\right) /(\rho-\alpha)\right]\right\}\left(1 / g\left(\bar{R}^{q}\right)\right)^{\theta}
$$

and

$$
\beta[(\theta-\gamma) /(\theta-1)]\left[P-\bar{R}^{q} /(\rho-\alpha)\right]\left(g\left(\bar{R}^{q}\right) / \bar{R}^{q}\right)^{\gamma}+\eta\left[g\left(\bar{R}^{q}\right) /(\rho-\alpha)\right]-[\theta /(\theta-1)] P=0
$$

\section{A.2.1 Nä̈ve beliefs}

Substituting $q=n, g\left(\bar{R}^{n}\right)=R^{n}$ and $\bar{R}^{n}=R^{*}$ into Eq. (A.2.6) and Eq. (A.2.7) yields:

$$
k=\left\{P-\beta\left[P-R^{*} /(\rho-\alpha)\right]\left(R^{n} / R^{*}\right)^{\gamma}-\eta\left[R^{n} /(\rho-\alpha)\right]\right\}\left(1 / R^{n}\right)^{\theta}
$$

and

$$
\beta[(\theta-\gamma) /(\theta-1)]\left[P-R^{*} /(\rho-\alpha)\right]\left(R^{n} / R^{*}\right)^{\gamma}+\eta\left[R^{n} /(\rho-\alpha)\right]-[\theta /(\theta-1)] P=0
$$

Plugging $k$ into Eq. (A.2.3) gives Eq. (8).

\section{A.2.2 Sophisticated beliefs}

Substituting $q=s$ and $\bar{R}^{n}=\widetilde{R}$ into Eq. (A.2.6) and Eq. (A.2.7) yields:

$$
k=\left\{P-\beta[P-\widetilde{R} /(\rho-\alpha)](g(\widetilde{R}) / \widetilde{R})^{\gamma}-\eta[g(\widetilde{R}) /(\rho-\alpha)]\right\}(1 / g(\widetilde{R}))^{\theta},
$$

and

$$
\beta[(\theta-\gamma) /(\theta-1)][P-\widetilde{R} /(\rho-\alpha)](g(\widetilde{R}) / \widetilde{R})^{\gamma}+\eta[g(\widetilde{R}) /(\rho-\alpha)]-[\theta /(\theta-1)] P=0
$$

Then, imposing the stationarity condition $g(\widetilde{R})=\widetilde{R}=R^{s}$ yields:

$$
k=\left\{P-\beta\left[P-R^{s} /(\rho-\alpha)\right]-\eta\left[R^{s} /(\rho-\alpha)\right]\right\}\left(1 / R^{s}\right)^{\theta},
$$

and

$$
R^{s}=\frac{\theta+\beta(\gamma-\theta)}{\eta(\theta-1)+\beta(\gamma-\theta)} P(\rho-\alpha)
$$

Plugging $k$ into Eq. (A.2.3) gives Eq. (11).

\section{A.3 Timing thresholds: Properties}

Define the function $Z(x)=\beta[(\theta-\gamma) /(\theta-1)]\left[P-R^{*} /(\rho-\alpha)\right]\left(x / R^{*}\right)^{\gamma}+\eta[x /(\rho-\alpha)]-[\theta /(\theta-1)] P$. Note that $Z(x)$ is convex in $x, Z\left(R^{n}\right)=0$ and $Z\left(R^{*}\right)<0$. Optimality requires that $Z^{\prime}\left(R^{n}\right)>0$. 
Hence, it follows that $R^{*}<R^{n}$. Then, as $\theta>\gamma$, it can easily be shown that $[\gamma /(\gamma-1)]<$ $[\theta+\beta(\gamma-\theta)] /[\eta(\theta-1)+\beta(\gamma-\theta)]<[\theta /(\theta-1)]$. This in turn implies that $R^{*}<R^{s}<R^{* *}$.

Finally, define the function $Z(x ; \widetilde{x})=\beta[(\theta-\gamma) /(\theta-1)][P-\widetilde{x} /(\rho-\alpha)](x / \widetilde{x})^{\gamma}+\eta[x /(\rho-\alpha)]$ $-[\theta /(\theta-1)] P$. Denote by $g(\widetilde{x})$ the solution of the equation $Z(g(\widetilde{x}) ; \widetilde{x})=0$. Totally differentiating with respect to $\widetilde{x}$ yields:

$$
\begin{gathered}
\beta[(\theta-\gamma) /(\theta-1)]\{-1 /(\rho-\alpha)+[P-\widetilde{x} /(\rho-\alpha)][(1 / g(\widetilde{x})) \partial g(\widetilde{x}) / \partial \widetilde{x}-(\gamma / \widetilde{x})]\}(g(\widetilde{x}) / \widetilde{x})^{\gamma} \\
+\eta(\partial g(\widetilde{x}) / \partial \widetilde{x}) /(\rho-\alpha)=0
\end{gathered}
$$

Rearranging gives:

$$
\partial g(\widetilde{x}) / \partial \widetilde{x}=\frac{\beta[(\theta-\gamma) /(\theta-1)](g(\widetilde{x}) / \widetilde{x})^{\gamma} \widetilde{x}(\gamma-1)}{\beta[(\theta-\gamma) /(\theta-1)][P(\rho-\alpha)-\widetilde{x}](g(\widetilde{x}) / \widetilde{x})^{\gamma-1}+\eta \widetilde{x}}\left(\frac{R^{*}}{\widetilde{x}}-1\right)<0, \text { for } R^{*}<\widetilde{x}
$$

Note that:

$$
Z\left(R^{n} ; R^{*}\right)=Z\left(R^{s} ; R^{s}\right)=0
$$

Hence, as $R^{*}<R^{s}$, it follows that $R^{n}<R^{s}$. 


\section{References}

[1] Capozza, D., Helsley, R.W., 1990. The stochastic city. Journal of Urban Economics, 28, 187-203.

[2] Capozza, D., Li, Y., 1994. The intensity and timing of investment: the case of land. American Economic Review 84, 889-904.

[3] Di Corato, L., 2012. Optimal conservation policy under imperfect intergenerational altruism. Journal of Forest Economics, 18, 3, 194-206.

[4] Dixit, A.K., Pindyck, R.S., 1994. Investment under uncertainty. Princeton University Press, Princeton, NJ.

[5] Frederick, S., Loewenstein, G., O’Donoghue T., 2002. Time discounting and time preference: a critical review. Journal of Economic Literature, 40, 2, 351-401.

[6] Geltner, D., Riddiough, T., Stojanovic, S., 1996. Insights on the effect of land use choice: the perpetual option on the best of two underlying assets. Journal of Urban Economics 39, 1, 20-50.

[7] Grenadier, S.R., Wang, N., 2007. Investment under uncertainty and time-inconsistent preferences. Journal of Financial Economics, 84, 2-39.

[8] Harris, C., Laibson, D., 2013. Instantaneous gratification. The Quarterly Journal of Economics, 128, 1, 205-248.

[9] Irwin, E.G., Bockstael, N.E., 2002. Interacting agents, spatial externalities and the evolution of residential land use patterns. Journal of Economic Geography, 2, 31-54.

[10] Loewenstein, G., Prelec, D., 1992. Anomalies in intertemporal choice: evidence and an interpretation. Quarterly Journal of Economics, 107, 573-598.

[11] Nishihara, M., 2012. Real option valuation of abandoned farmland. Review of Financial Economics, 21, 4, 188-192.

[12] O’Donoghue, T., Rabin M., 1999. Doing it now or later. American Economic Review, 89, 103-124.

[13] Pollak, R.A., 1968. Consistent planning. Review of Economic Studies 35, 2, 201-208.

[14] Salois, M.J., Moss C.B., 2011. A direct test of hyperbolic discounting using market asset data. Economics Letters, 112, 290-292.

[15] Salois, M.J., 2012. The effect of quasi-hyperbolic discounting on rural land conversion 
timing. Applied Economics Letters, 19, 887-890.

[16] Schatzki, T., 2003. Options, uncertainty and sunk costs: an empirical analysis of land use change. Journal of Environmental Economics and Management, 46, 86-105.

[17] Song, F., Zhao, J., Swinton, S.M., 2011. Switching to perennial energy crops under uncertainty and costly reversibility. American Journal of Agricultural Economics, 93, 3, 768-783.

[18] Strotz, R.H., 1956. Myopia and inconsistency in dynamic utility maximization. Review of Economic Studies, 23, 165-180. 


Sveriges lantbruksuniversitet

Swedish University of Agricultural Sciences

Department of Economics

Department of Economics

Swedish University of Agricultural Sciences (SLU)

P.O. Box 7013, SE-750 07 Uppsala, Sweden

Ph. +4618 671000

www.slu.se

www.slu.se/economics
Institutionen för ekonomi

Sveriges lantbruksuniversitet

Box 7013, 75007 Uppsala

Tel. 018-67 1000

www.slu.se

www.slu.se/ekonomi 